\title{
Molecular Historiography - neue Gegenstände und neue Methoden einer neuen Geschichtsschreibung?
}

Alfons Labisch

Staffan Müller-Wille und Christina Brandt (Hg.) 2016: Heredity Explored. Between Public Domain and Experimental Science, 1850-1930. Boston: The MIT Press, geb., 480 S., $€ 40,59$, ISBN: 9780-262-03443-2.

Marianne Sommer 2016: History Within. The Science, Culture, and Politics of Bones, Organisms, and Molecules. Chicago: The University of Chicago Press, geb., 496 S., $€ 41,39$, ISBN: 978-0-22634732-5.

Elsbeth Bösl 2017a: Doing Ancient DNA. Zur Wissenschaftsgeschichte der aDNA-Forschung. Bielefeld: Transcript, 460 S., $€ 39,99$, ISBN: 978-383-763900-1.

Stefanie Samida, Jörg Feuchter (Hg.) 2016: The Genetic Challenge to Medieval History and Archaeology: Medieval Worlds (4) https://doi.org/10.1553/medievalworlds_no4_2016, ISSN: 2412-3196, ISBN-13 Online: 978-3-7001-8084-5, URL: http://www.medievalworlds. net/8084-5inhalt

Sind wir auf dem Weg zu einer molecular historiography, zu einer molekularen Geschichtsschreibung? Dass sich diese Frage überhaupt stellt, hängt mit den faszinierenden Ergebnissen zusammen, die die Archäologie mit modernsten natur- und lebenswissenschaftlichen bzw. molekularbio- 
logischen Methoden für die Frühzeit der Menschheitsgeschichte erbringt. Der Mann vom Tisenjoch, volkstümlich Ötzi genannt, der Bogenschütze von Amesbury oder das Mädchen von Egtved - um einige Beispiele zu nennen - haben allgemeines Interesse gefunden: Die Öffentlichkeit reagiert fasziniert auf derartige Funde. Was wir über die Lebensumstände der Menschen, die 5.000 Jahre vor unserer Zeit lebten, wissen, ist mit modernen naturwissenschaftlichen und biologischen Methoden ermittelt worden.

Molecular historiography heißt, dass sämtliche Möglichkeiten und Interaktionen zwischen Mensch und Umwelt Gegenstand historischer Erkenntnis - daher historiography - sein können, sofern entsprechendes genetisches Material gefunden, aufbereitet und ausgewertet werden kann. Der geläufige Begriff genetic history erfasst, wie die molekulare Transition der Medizin zeigt (Labisch 2004), die Möglichkeiten einer molecular historiography nur teilweise.

Was wissen wir über die Menschheit vor der Zeit der Schriftlichkeit? Das ist eine elementare Frage. Beruht die Geschichtsschreibung ausschließlich auf schriftlichen Quellen? Nein, gewiss nicht. War dies schon zuvor nicht der Fall, so sind in den letzten Jahrzehnten viele weitere Gegenstände historischer Forschung aufgedeckt worden. Aber jetzt geschieht etwas Anderes - Gegenstände und Methoden weiten sich auf den „bios“, auf die Natur und die gesamte natürliche Umwelt des Menschseins, und damit auch auf materiale Gegebenheiten und Umstände menschlichen Lebens insgesamt aus. ${ }^{1}$

Damit können einige Fragen für die nachfolgenden Gedanken formuliert werden:

- Welche Lebens- und Erfahrungsbereiche sind mögliche Gegenstände molekular-historiographischer Untersuchung?

- Wie sind die molekularbiologischen bzw. naturwissenschaftlich-technischen Methoden zu bewerten, mit denen historiographische Aussagen erzielt werden?

- Welche Aussagen lassen molekular-historiographische und naturwissenschaftliche Methoden zu?

- Was sind in einem Analogieschluss zur quellenkritischen Textanalyse die Kriterien der inneren und äußeren Quellenkritik für sämtliche Zeugnisse menschlicher Existenz?

- Welche Kontexte können mit den jeweiligen Quellen erfasst werden?

- Welche Kontingenzen und damit welche besonderen Ereignisse, Handlungen und Zufälle - ein Spezifikum historischer Forschung - können mit den jeweiligen Quellen erfasst werden? 
- Welche allgemeinsten Anforderungen an eine molekulare Historiographie resultieren aus diesen Überlegungen?

Diese für die Historiographie fundamentalen Fragen können in diesem Essay nur angedeutet werden. Dies gilt umso mehr, als sich die nachfolgenden Gedanken nur auf einige wenige Publikationen aus der inzwischen recht breiten Literatur zum Thema stützt. Es trifft sich daher gut, dass sowohl in dieser Zeitschrift als auch andernorts eine breiter angelegte Diskussion zum Thema erfolgen soll. ${ }^{2}$

Soll die Kooperation zwischen Historikern, Archäologen und Molekularhistorikern bedacht erfolgen - und eben darum geht es -, gilt es das Gebiet, die Gegenstände und die Methoden des anderen Fachgebietes zu kennen. Da bestimmte Methoden ggf. ausschließlich im Gebiet der Naturund Lebenswissenschaften vorkommen, sind sie in der Alltagswelt genuiner Geisteswissenschaftler üblicherweise nicht vertreten. Hier sind also rein technische Unkenntnis und Missverständnisse samt entsprechender Unter- wie Überinterpretationen vorprogrammiert. Umgekehrt gilt selbstredend das Gleiche: Ein genuines Verständnis für geisteswissenschaftliche, speziell historiographische Forschungen habe ich unter Natur- und Lebenswissenschaftlern zwar durchaus, aber nur selten vorgefunden. Mit Blick auf die molecular historiography ist zu fragen, wie entsprechende Gebiete entstanden, welche Gegenstände und Methoden sich herauskristallisiert haben, wie die gewonnenen Ergebnisse einzuschätzen sind.

Auch dies sei vorweg betont: Das Genom eines Menschen ist das höchst individuelle biologische Archiv seines Lebens, das ggf. durch weitere naturwissenschaftliche Daten - etwa über seine Lebensumstände und Lebenswege - zu einem Biom seines gesamten Lebens ergänzt werden kann. Dies geschieht allerdings, ohne über das Denken und Handeln dieses Menschen und die vielen Zufälligkeiten eines menschlichen Lebens, die aus dem $\mathrm{Zu}$ sammenleben erwachsen, auch nur das Geringste aussagen zu können. Eben hier liegt das klassische Gebiet der Geschichtsschreibung.

Die Auswahl der besprochenen Bücher umfasst die ganze Bandbreite vom Allgemeinen zum Speziellen. Der Sammelband von Staffan MüllerWille und Christina Brandt liefert einen Überblick über die Genese der Genetik. Marianne Sommer zeigt an drei Beispielen, wie die Genetik nach und nach andere Wissenschaften infizierte und letztlich in die Lebenswelt diffundierte. Elsbeth Bösl stellt am Beispiel von aDNA ebenso umfassend wie kritisch die Forschungsgeschichte und den Stand der molecular historiography dar. Und schliesslich gehen Samida und Feuchter die Probleme und Fragen der angewandten molekularhistorischen Forschung unmittelbar an. 
Wie ist das Gen in unsere Welt getreten? Und vor allem: Mit welchen gesellschaftlichen Konnotationen und Wirkungen ist dies geschehen? Denn es ist allseits offenkundig, dass die Vererbungslehre und ihre Substrate außerordentlich weitreichende gesellschaftliche und historische Implikationen haben, die in der aktuellen Diskussion einer molecular historiography durchschlagen. Hier ist der von Staffan Müller-Wille und Christina Brandt herausgegebene Band Heredity Explored. Between Public Domain and Experimental Science, 1850-1930 zu nennen.

Unter dem Titel Genealogy, Kinship, and Population werden Literaturwissenschaften, Verwandtschaftsehen, Asyle und staatliche Effizienz sowie die Frage sozialer Emanzipation am Beispiel der jüdischen Integration in Deutschland bis in die dreißiger Jahre des vorigen Jahrhunderts behandelt. Im Kapitel Heridity, Evolution, and Reproduction werden die Fragen der Erblichkeit vor der Genetik, natürliche Selektion und Erblichkeit im Darwinschen und Nach-Darwinschen Denken und Fragen der Geschlechtsunterschiede in der Genetik angesprochen. Unter dem Aspekt Heredity in Agro-Cultural Contexts geht es um genetisch reine Linien, um die Entwicklung von Impfstoffen und die Frage der Patentierung als Interaktion von Erbschaftsforschung und Erfindungen. Heredity in Medical Contexts vereint Aufsätze zur Erfindung der Pränatalität, die fragliche Transmission der Frage der Erblichkeit in die Pathologie des Menschen und die Suche nach der idealen Bevölkerung. Das letzte Kapitel ist schließlich dem Mendelism gewidmet: die Ursprünge der Genetik, die Ausbreitung der Erblichkeitslehre über den Atlantik und die Frage nach "reinen Linien“ in Laboratorien.

Herausgehoben sei die Studie von Veronica Lipphardt über die angeblich emanzipatorische Kraft der Erblichkeit anhand früher Forschungen zur genetischen Eigenart der Juden (S. 111-139). Juden scheinen aus vielerlei Sicht ein herausragendes Beispiel für genetische Untersuchungen zu sein: Isolation, Endogamie, Vererbung von Eigenschaften, alles dies kommt hier gleichsam im historischen Experiment zusammen. In diesem Sinne haben auch jüdische Forscher, dargestellt an Wilhelm Nussbaum, versucht, die Richtigkeit der Erbgesetze an großen jüdischen Gruppen zu prüfen, um daraus emanzipatorische Argumente abzuleiten. Aber, so schlussfolgern zuvor bereits Herausgeberin und Herausgeber, es gibt keinen direkten Zusammenhang zwischen biologischer Erblichkeit und politischen Überzeugungen (S. 8). In diesem Zusammenhang ist anzumerken, dass in der aktuellen genetischen Forschung dieselben Fragen wiederholt werden. Denn eine zunehmende Zahl von Wissenschaftlergruppen versucht mit beeindruckendem Aufwand, die Tatsache und die Eigenart eine ,jüdischen Genoms" herauszuarbeiten (Atzmon et al. 2010; Behar et al. 2010). Und ähnlich wie schon in den 1930er Jahren ziehen auch diese Studien in der 
internationalen Kritik alle jene Gegenreden auf sich, die bei solchen lebensweltlich intentional überhöhten Studien notwendig getroffen werden. Was ist - so ein Standardeinwurf - eigentlich das aktuelle Vergleichssample, aus dem heraus so etwas wie ein historisches jüdisches Genom nachgewiesen werden kann, das eine Population repräsentieren soll, die sich seit etwa 2.500 Jahren vor unserer Zeit über die Erde verbreitet hat? Ist die Frage des Judentums letztlich eine biologische Frage oder eine Frage von Tradition und Kultur (Ostrer \& Skorecki 2013)?

Auch den Beitrag von Christophe Bonneuil über Pure Lines as Industrial Simulacrea: A Cultural History of Genetics from Darwin to Johannsen (S. 213-242) möchte ich herausheben: Willem Johannsen schöpfte seinen säkularen Gedanken, dass Mendels Stoff, der letztlich Erblichkeit vermittle, in dem Konzept "Gen“ zu fassen sei, aus dem Geist des reinen Bieres! Carlsberg - der bekannten dänischen Brauerei - und deren Versuch, genetisch reine Hefelinien in ihre Produktion einzuführen, sei Dank, gibt es seit 1909 die so überaus einflussreiche theoretische Unterscheidung von Genotyp und Phänotyp. Und hier sind wir wieder bei den „wissenschaftlichen Wahrheiten“: Über die Jahre in den Chromosomen als feste biologische Entitäten ausgemacht, in ihrer Struktur erkannt und schließlich in ihre verschiedenen Bestandteile und deren mögliche Funktionen zerlegt, ist das genetische Gesetz des ausgehenden 20. Jahrhunderts „Ein Gen, ein Protein - ein Protein, ein Gen“" mittlerweile obsolet. Die einstmals diskussionsbeherrschenden Gene sind in den jüngsten molekularbiologischen Konzepten zu einer Art Blaupause zahlreicher Proteingebilde geschrumpft. Deren Funktion ist weniger aus den Genen als aus der Gen-Umwelt-Interaktion zu erklären. Die Epigenetik, über Jahrzehnte marginalisiert, steht heute in der vorderen Linie molekularbiologischer Forschung. Und über die Epigenetik wandert notwendig die allgemeine Geschichtsschreibung in die molecular historiography ein.

Den Herausgebern geht es darum, die Frage der Erblichkeit in einen breiten Horizont kultureller, sozialer, politischer und wissenschaftlicher Diskurse zu setzen. Nur auf diese Weise wird deutlich, was mit den Begriffen auch dann gemeint wird, wenn sie sich in den scheinbar neutralen Hafen der Wissenschaften geflüchtet haben. Angesichts der Zahl von Autoren und Themen liegt der schlichte Vorwurf nahe, in diesem Sammelband sei keine einheitliche Linie zu erkennen. Nun ist es den Herausgebern prima vista gar nicht um diese Linie gegangen. Ihnen ging es vielmehr darum aufzuzeigen, auf welcher Vielfalt historischer Hintergründe das Generalthema der Erblichkeit die verschiedensten Wissensbereiche infizieren und schließlich in die Gesellschaft diffundieren konnte.

In denselben Themenkreis gehört die Monographie von Marianne Sommer. An drei Beispielen - Henry Fairfield Osborn (1857-1935), Julian Sorell 
Huxley (1887-1975) und Luigi Cavalli-Sforza - zeigt Marianne Sommer, wie die Erblichkeitslehre sich mehr und mehr auf die Genetik hinbewegte, auf diesem Weg immer weitere Wissensgebiete infizierte und schließlich über eine gezielte Öffentlichkeitsarbeit als eine Art genetischer Kultur in die Gesellschaft diffundierte.

Osborn war Präsident des 1869 gegründeten American Museum of Natural History, das er in einen weltweit vernetzten Ort paläoontologischer und paläoanthropologischer Forschung verwandelte. Das auf Rasse und Geschlecht beruhende Weltbild Osborns schlug letztlich auf die von Millionen von Menschen besuchten Ausstellungen des Museums durch. Huxley war von 1935 bis 1942 Direktor des 1823 gegründeten London Zoo, erster Generalsekretär der UNESCO (1946-1948) und Mitbegründer des World Wildlife Fund (1960). Er nutzte seine Einrichtungen und Mittel, um aus Sicht einer evolutionären Synthese für einen wissenschaftlichen Humanismus zu sprechen. Es ging ihm um das Verständnis der Genese der Umwelt und der Lebewesen einschließlich der Menschen, um auf der Basis dieses Wissens gegen die Destruktivität der Menschen zu argumentieren. Mitten in das Zentrum einer molecular historiography führt der seit 1970 in Stanford lehrende italienische Genetiker Luigi Cavalli-Sforza mit dem ersten weltweiten genetisch-historischen Großprojekt. Aus der Analyse zunächst serologischen, dann genetischen Materials erstellte CavalliSforza einen Atlas der Verteilung der Menschen und ihrer verschiedenen Gruppierungen über die gesamte Erde: So entstand eine Weltkarte der Wanderbewegungen des homo sapiens.

Cavalli-Sforza ist mit seiner Frage, ob die Gene, deren Varianz und Verteilung auch etwas über die Geschichte der Menschheit aussagen, einer der Mitbegründer der molekularen bzw. genetischen Anthropologie geworden. Er verband mithilfe der seinerzeit allmählich verfügbaren elektronischen Rechenmaschinen biologische Daten mit vorhandenen demographischen, linguistischen, archivalischen und archäologischen Daten: zunächst mit Blutgruppen, später mit genetischen Merkmalen, darunter vor allem der mitochondrialen DNA und damit zunächst der matrilinearen Vererbung. Er konnte sich damit gleich zwei elementare Neuerungen zu eigen machen - die elektronische Datenverarbeitung und die molekulare Genetik, die ihrerseits wiederum essentiell von der elektronischen Datenverarbeitung abhängig und in diesem Sinne eine technikgetriebene Disziplin ist. Mit diesem Verbund unterschiedlicher Quellen hat Cavalli-Sforza zugleich die methodischen Standards gesetzt. In seinen Arbeiten wandte er sich stets dezidiert dagegen, Menschen in Rassen einzuteilen. Vielmehr sah er in der genetischen Varianz Reaktionen auf geänderte Umweltbedingungen und unterschied letztlich Gruppen von menschlichen Populationen, die jeweils durch unterschiedliche genetische Marker gekennzeichnet sind. Cavalli- 
Sforza betont, dass die genetische Varianz von Individuen innerhalb einer Population größer ist als die zwischen Populationen. Die augenfälligen äußerlichen „Rassen“-Merkmale physischer Andersartigkeit stimmen mit der genetischen Varianz nicht überein. Das bedeutet, dass der Begriff „Rasse“ und die daraus resultierenden sozialen und politischen Schlussfolgerungen weniger biologische als kulturell-historische Konstrukte sind.

Sommer geht es nicht, wie auf den ersten Blick zu befürchten, um die Biographien ihrer drei Protagonisten. Den Hintergrund bilden vielmehr jeweils unterschiedliche Forschungsprogramme, institutionelle Vorgaben, verschiedene Objekte - Fossilien, Knochen und Gene - sowie unterschiedliche Ideen von der Genese der Menschheit und verschiedener Popularisierungsformen samt der unterliegenden politischen Grundanschauungen. Vor diesen Kulissen arbeitetet Sommer an der Lebensarbeit drei verschiedener Wissenschaftler heraus, wie sowohl die wissenschaftliche Welt als auch die allgemeine Lebenswelt von den jeweiligen Konzepten der Erblichkeit durchdrungen und beeinflusst worden sind. Im Fokus von Sommers Buch steht damit im Verbund individueller Wissenschaftlerbiographien und vielfältiger äußerer Bedingungen die öffentliche Dimension der Erblichkeitslehre - von den Wissenschaften über die Geschichtsschreibung bis hin zu Literatur und Künsten werden die entsprechenden Aussagen rezipiert und damit letztlich in der Weltwahrnehmung einer breiten Öffentlichkeit wirksam. Die „molekulare Eva“ als Urmutter des homo sapiens oder die ggf. nur aus wenigen Knochen rekonstruierten Dinosaurier sind Ikonen des späten 20. Jahrhunderts geworden.

In das Zentrum der Diskussion um eine molecular historiography führt Elsbeth Bösls Buch Doing Ancient DNA. Zur Wissenschaftsgeschichte der aDNA-Forschung. ${ }^{3}$ Die Tatsache, dass die molekulare Historiographie mitten in der historischen Forschung angelangt ist und dort $\mathrm{zu}$ erheblichen Diskussionen geführt hat, ist die allgemeine Problemstellung Bösls:

Wie betreiben GenetikerInnen oder BiochemikerInnen Geschichtsforschung? Was meinen sie, wenn sie von DNAMolekülen als Quellen, Archiv oder Wissensspeicher sprechen? Wächst da eine Geschichtskonkurrenz heran, die mit alter DNA und stabilen Isotopen die Quellen und Deutungen der HistorikerInnen überbieten will? Lassen sich biologische Daten überhaupt soziokulturell interpretieren? Ist es zulässig, zum Beispiel Haplotypenverteilungen mit Populationen, geografischen Räumen oder sogar archäologischen Kulturen, also Verbreitungen bestimmter Sachguttypen, in Verbindung zu bringen? Droht hier nicht Biologismus und gerät nicht der mühsam erkämpfte Konstruktivismus der Geschichtswissenschaften in Gefahr? (Bösl 2017a: 99) 
So oder ähnlich lauten die Fragen seitens der Historiographie. Die spezielle Problemstellung von Bösls eigenem opus magnum ist zunächst die Frage, was denn ancient DNA ist: Wie hat sich dieses molekulargenetische Substrat der molecular historiography rein wissenschaftshistorisch herausgebildet? Daran schließen sich notwendig die Fragen an, aufgrund welcher biologischen Tatsachen Molekularbiologen und Genetiker zu historischen Aussagen kommen und dabei tief in das Feld der Archäologie, der Geistesund der Kulturwissenschaften vordringen. Zugleich ist es Bösls Absicht, die methodischen Verfahren der molecular historiography für Geistes- und Kulturwissenschaftler verständlich zu machen.

Bösl baut ihre Monographie systematisch auf, zunächst werden Problem- und Fragestellung expliziert. Im Nachfolgenden wird über den Forschungsstand zu aDNA-Research und über die Entstehung des Forschungsfeldes berichtet. Ausführlich werden die erheblichen technischen Rückschläge herausgearbeitet: Hier wird deutlich, dass uns mit der molecular historiography keine naturwissenschaftlich-technische Disziplin entgegentritt, die ihre ggf. apodiktischen Urteile gleichsam aus der beständigen Struktur des Forschungsgegenstandes, aus der technischen Faktizität ihrer Maschinerie und den daraus beliebig wiederholbaren Produkten schöpfen kann. Schließlich behandelt Bösl die Anwendungen und Fragestellungen, die mithilfe der aDNA über die vergangenen nahezu vierzig Jahre als möglich angesehen wurden und unterzieht sie einer Kritik. Diese Kritik stützt Bösl auf ihre umfassende Kenntnis der technischen Grundlagen der aDNA-Forschung. Doing ancient DNA - das steht im Zentrum von Bösls Untersuchung: Wie wird eine Quelle wissenschaftlich hergestellt? Damit wird zugleich der elementare Unterschied zu den klassischen historischen Quellen deutlich: aDNA wird üblicherweise nicht in verwertbarer Weise vorgefunden, wie bei Überresten etwa in der klassischen Archäologie oder in der Geschichtsforschung üblich. Vielmehr wird die Quelle durch aufwendige technische Verfahren überhaupt erst produziert. Diese Verfahren sind, da sie stets nur einen ggf. winzigen Ausschnitt der Gesamtsituation darstellen, notwendig Teil der Quelle bzw. ihrer Aussagemöglichkeiten. Dabei sind keineswegs nur apparative Fragen, sondern auch Fragen des gesamten Studiendesigns, des Samples und der Vergleichspopulationen und damit statistische Fragen - wichtig.

Gleichwohl sind die Ergebnisse der molecular historiography faszinierend. Denn es werden neue Felder aufgetan, die einen Blick in die Geschichte der Menschheit insbesondere jenseits der Schriftlichkeit ermöglichen. Hier verweist Bösl auf das ancient microbiom als Gesamtheit aller genetischer Materialien, die sich um einen Fund gruppieren lassen. Es können damit also nicht nur Individuen und Gruppen in ihrer zeitlichen und 
räumlichen Entwicklung, sondern ggf. auch ihre Lebensumstände erfasst werden. Dies ist nicht genetic history, sondern molecular historiography.

Unter dem Generalsatz „Der Wert einer Quelle ist nicht absolut, sondern hängt von der Problemstellung ab“ kommt Bösl zu folgendem Schluss:

DNA erwies sich als in bestimmten Graden ebenso interpretativ flexibel wie andere Quellen, und genauso hatte sie ein Vetorecht wie andere Quellen. Die Informationen, die sich aus ihr generieren ließen, waren nicht wichtiger oder gewisser, sondern meist nur anders als die aus anderen Quellen gewonnenen Erkenntnisse, da unterschiedliche Quellenarten auch unterschiedliche Aspekte der Vergangenheit repräsentieren. (...) Anerkennen mussten sie (d.i.: Genetiker, Prähistoriker, Paläoanthropologen und Paläoepidemiologen; sc. AL), dass Quellen je nach Fragestellung unterschiedlichen Wert haben und zum Beispiel molekulare Quellen nicht per se mehr oder gewisseres, sondern schlicht anderes Wissen ermöglichen als archäologisches Sachgut. (Bösl 2017a: 347)

Dies festzustellen bedeutet, dass sich letztlich eine kritisch-konstruktive Zusammenarbeit zwischen den verschiedenen Disziplinen herauskristallisieren wird, die sich nach den jeweils anliegenden Problem- und Fragestellungen richtet.

Elsbeth Bösl schließt ihr Buch mit einem umfangreichen, nahezu 100 Seiten umfassenden Quellen- und Literaturverzeichnis (353-449) und einem Register (455-458) ab - ein unabdingbares Instrument, um diese umfangreiche und bis tief in die Einzelheiten auch der biologisch-technischen Entwicklung gehende Arbeit auszuschöpfen. Elsbeth Bösl hat mit ihrem Werk einen Meilenstein in der Diskussion zwischen klassischer Historiographie und molecular historiography gesetzt: Wer immer sich in deutscher Sprache nicht nur über die methodische, technische und historiographische Genese des Gebietes, sondern auch über die Auseinandersetzungen sowohl innerhalb der beteiligten biologischen Fachgebiete als auch zwischen molecular historiography und den klassischen Disziplinen Archäologie, Ur- und Frühgeschichte, Geschichtswissenschaften generell und Kulturgeschichte informieren möchte, kommt an diesem Werk nicht vorbei.

Die vierte hier vorgestellte Publikation führt direkt hinein in die Diskussion um die Vor- und Nachteile einer molecular historiography und die Möglichkeiten für bzw. Widerstände gegen eine Kooperation zwischen den natur- und lebenswissenschaftlichen Disziplinen einerseits und den geschichts- und kulturwissenschaftlichen Disziplinen andererseits. Das Themenhaft der Medieval Worlds (2016) versammelt einige Beiträge zu einer Konferenz, die Jörg Feuchter und Stefanie Samida 2015 an der Berliner Humboldt-Universität organisiert haben. 
Sebastian Brather verweist in seinem Beitrag New Questions instead of Old Answers:

Archaeological Expectations of aDNA Analysis auf die unterschiedlichen Konstrukte in den beteiligten Disziplinen: Gruppen, Ethnien, Nationen sind immer soziale und kulturelle Konstrukte, die aus der jeweiligen historischen Situation stammen. Ancient DNA und andere biologische Substrate grenzen hingegen biologisch definierte Populationen ab. Diese stimmen nur bedingt mit den historischen Fremd- und Selbstdefinitionen überein. Die biologischen Ergebnisse mit den kulturhistorischen Ergebnissen gleichzusetzen, wäre wissenschaftlich ein Rückfall in das simple Genotyp-gleichPhänotyp-Denken des 19. Jahrhunderts. Allerdings verspricht die komplexe Interaktion zwischen den unterschiedlichen Disziplinen neue Perspektiven, die nur dann verfolgt werden können, wenn historische und biologische Disziplinen eng zusammenarbeiten. Es geht also letztlich darum, gemeinsam neue Fragestellungen zu erarbeiten.

Die weiteren Beiträge des Bandes kreisen aus Sicht der Geistes- und Kulturwissenschaften bzw. der Natur- und Lebenswissenschaften beispielhaft um migrationshistorische Studien und damit um ein elementares Thema molekularhistorischer Forschung. Stefan Burmeister behandelt die grundsätzliche Frage der Archaeological Research on Migration as a Multidisciplinary Challenge. Zwar ist Migration ein Schlüsselkonzept der Archäologie. Aber Migration vorauszusetzen, um die Verteilung kultureller Grundmuster zu erklären, sei inzwischen obsolet, so Burmeister, da geschriebene Quellen, die Archäologie und die neuen molekularbiologischen Methoden einander korrigierten. In dem Beitrag Mapping European Population Movement through Genomic Research analysieren Patrick J. Geary und Krishna Veeramah moderne populationsgenetische Studien über Migrationsbewegungen im Europa vom vierten bis neunten Jahrhundert unserer Zeit. Die Autoren verweisen auf neue Studien, die sich auf das gesamte Genom stützen. Sich mit aDNA zu befassen, setzt allerdings voraus, Archäologen und Historiker in die Studiengruppen zu integrieren, um die Befunde umfassend interpretieren zu können. Manfred K.H. Eggert diskutiert an der Frage Geneticizing Bantu: Historical Insight or Historical Trilemma? die unterschiedlichen Forschungsansätze der Linguistik am Beispiel der Bantu-Sprache (das europäische Beispiel wäre die „vaskonische Hypothese“ aus der Linguistik), der Bantu-Archäologie und der Bantu-Genetik. Kerstin P. Hofmann problematisiert am Beispiel der Besiedlung Englands die Frage von genetischem Material und Identität: With víkingr into the Identity Trap: When Historiographical Actors get a Life of their Own. Gibt es genetisches Material, dass auf Wikinger im Genpool der britischen Bevölkerung hinweist? Wer ist, was bedeutet überhaupt Wikinger? Stimmt die vielfach überlagerte Semantik dieses Begriffes mit dem überein, was archäo- 
logische und genetische Funde und Interpretationen überhaupt aussagen können? Hofmann analysiert und problematisiert die zentralen Konzepte Zeit, Raum, Mobilität und vor allem Identität jeweils aus der Sicht der Archäologie und der historischen Genetik. Hofmann plädiert energisch dagegen, im Streit der Fächer archäologische Subjekte zu „essentialisieren“ (S. 91, 103, 104) bzw. historiographische Narrative und Akteure zu naturalisieren. In ihrem forschungshistorisch orientierten Beitrag Genetic History and Identity: The Case of Turkey kommt Celine Wawruschka zu ähnlichen Ergebnissen: der zentralasiatische Beitrag zum Genpool der Türken bleibt in den bisherigen Publikationen offen.

Das Ergebnis dieses Bandes ist bereits im Editorial Why Archaeologists, Historians and Geneticists Should Work Together - and How vorgegeben: Es geht nicht um Konfrontation, es geht um Kooperation. Denn einerseits hat die molekulare Genetik einen völlig neuen Ansatz und völlig neue Ergebnisse für die Geschichte der Menschheit eröffnet, andererseits muss die Kooperation von der jeweiligen Kenntnis der methodischen Möglichkeiten und der Reichweite der möglichen Aussagen einer Disziplin gekennzeichnet sein.

\section{Schlussfolgerungen}

Die Haupt- und Nebengedanken, die bislang angeklungen sind, möchte ich abschließend versuchen, in einem Vergleich zusammenzufassen und zugleich den eingangs formulierten Katalog von Fragen aufrufen. Der Standpunkt, von dem aus dieser Vergleich gezogen wird, ist die Geschichte und Theorie der Medizin. Dieses Fach der Medizinischen Fakultät hat gleichermaßen die Geschichtswissenschaften wie die Medizin als Bezugsdisziplinen. Über die Medizin ist die Medizingeschichte mit den neuesten Entwicklungen in den molekularen Lebenswissenschaften verbunden. Und wenn in den nachfolgenden Zeilen einige holzschnittartige oder auch triviale Aussagen getroffen werden, ist das dem Bestreben geschuldet, zu einem möglichst einfachen und klaren Vergleich zu kommen, der eventuell als didaktisches Hilfsmittel dienen mag.

Wir Menschen können unsere Gegenwart nicht in ihrer Gesamtheit erfassen. Das heißt zugleich: Einen Zugang zur Vergangenheit in ihrer Gesamtheit kann es nicht geben. Alle historischen Betrachtungen liefern jeweils begrenzte Interpretationen von Ausschnitten vergangener Zeiten. Wenn es darum geht, ein möglichst umfassendes Bild zu erhalten, sind klare fachspezifische Fragestellungen und Methoden ebenso essentiell wie eine zwischen den Disziplinen abgestimmte Kooperation. 
Gegenstand der Geschichtswissenschaften ist das Handeln der Menschen in ihren zeitlich gegebenen Handlungsräumen: Individuelles und gemeinschaftliches menschliches Handeln bedeuten Kontingenz, Handlungsräume bedeuten Kontext. Wesentliche Aufgabe und damit wesentliches Merkmal der Geschichtsschreibung ist, menschliches Handeln in seiner Sinnhaftigkeit und in seiner Kontingenz zu verstehen und mit Bezug auf den jeweils gegebenen Handlungsraum und die daraus resultierenden Handlungsoptionen zu erklären. Gegenüber allen jeweils in ihren sozialen Räumen legitimen Formen des Erinnerns zeichnet sich die professionelle Historiographie durch ihren unabdingbaren Bezug zu Überresten menschlichen Handelns aus. Diese Überreste sind grundsätzlich sämtliche Befunde, die auf die Existenz und das Handeln von Menschen verweisen. Die Fragestellung gibt an, mit welchem Erkenntnisinteresse und in welche Blickrichtung die Analyse der Überrestbefunde in ihrem zeitgerechten Kontext zu erfolgen hat: Erst durch die Problem- und Fragestellung wird ein beliebiger Überrest, ein beliebiger Befund zu einer Quelle - eine „Quelle an sich“ sagt gar nichts: Geschichtsschreibung ist problemgetrieben. Sollten keine Überreste vorhanden sein, ist keine valide Aussage möglich; sollten die Überreste gar den vermuteten Interpretationen widersprechen, ist die Aussage zu ändern: Es ist dies das Vetorecht der Quellen. Es sind grundsätzlich alle Quellen zu nutzen, eine etwaige Auswahl ist nachvollziehbar zu begründen. Die Quellen sind einer fachgerechten äußeren und inneren Quellenkritik zu unterziehen. Die äußere Quellenkritik richtet sich auf die physischen Eigenarten der Quelle, die innere Quellenkritik auf die Sinnhaftigkeit und Plausibilität der Botschaften, die den Quellen entnommen werden können. Die aus der Quellenkritik resultierenden Befunde sind in den zeitgerechten Kontext und die sich daraus ergebenden Handlungsoptionen einzuordnen. In diesem rekonstruierten Handlungs- und Entscheidungsraum können historische Ereignisse oder historische Entscheidungen jeweils mit Bezug auf die Überreste verstanden und erklärt werden. Durch Fragestellung, Befunde und historischen Kontext ergeben sich schließlich die Anhaltspunkte für eine historische Erzählung. Dieses Narrativ wird in unterschiedlichen Publikationsformen der Fachöffentlichkeit mitgeteilt. Aus der Wahrnehmung, Kritik und Diskussion der Fachöffentlichkeit ergeben sich letztlich so etwas wie „historische Tatsachen“. Diese Tatsachen unterliegen generell der Revision durch neue empirische Befunde und/oder neue, immer indes empirisch belegte Interpretationen: Die Historiographie ist eine genuin empirische Wissenschaft.

Diese holzschnittartige Umschreibung von Gegenstand und Methoden der Geschichtsschreibung sei nun der molecular historiography gegenübergestellt. Gegenstand einer molecular historiography sind biologisch-natürliche Handlungsräume und Überreste von Gruppen und Individuen, die 
ihrerseits ebenfalls biologisch definiert sind. Primär beschäftigt sich die molecular historiography mit Überresten, die sich weniger aus dem individuellen Handeln als aus dem „bios“ der Menschen und ihrer Umwelt ergeben. Die jeweils primären Fundmaterialien sind naturwissenschaftlich- oder lebenswissenschaftlich-technisch aufzubereiten. Dieser Aufbereitungsprozess ist erheblich von den Zufälligkeiten von Funden, von den Fundumständen, der Fundbearbeitung, von den angewandten Methoden und der maschinellen Ausstattung abhängig und damit technikgetrieben. Die Techniken befinden sich in einem rasanten Innovationsprozess.

Die Gegenstände der molecular historiography werden im Forschungsprozess erst erzeugt. Es handelt sich also um epistemische Dinge (Rheinberger 2001), die in einen bestimmten Erkenntniszusammenhang eingebunden sind und keine Objekte per se darstellen. Selbst bei einem microbiom, also bei einem umfassend gegebenen biologischen Material, können immer nur allgemeine Handlungsräume oder individuelle Lebensumstände analysiert werden. Menschliches Handeln in allen seinen Bedingungen und Zufällen wird mit Verfahren der molecular historiography nicht erfasst. Dies verdeutlicht der molekularbiologische Einsatz in klar definierten forensisch-rechtsmedizinischen Fragen. Auch in Rechtsfragen können nur die biologischen Tatbestände, keineswegs die persönlichen Hintergründe und Motive einer Handlung erklärt werden: Dies ist Aufgabe anderer Gutachter und des gesamten Gerichtsverfahrens.

Analog zur Gerichtsfestigkeit in der Forensik müssen die durch die natur- und lebenswissenschaftlichen Verfahren produzierten deutbaren Überreste einer strengen äußeren Quellenkritik unterzogen werden: Fundort, Fundumstände, technische Aufbereitung, mögliche Fehlerquellen von Fundumständen und Aufbereitungsformen etc. sind zu bedenken. Bei der inneren Quellenkritik gilt es, das gesamte Design einer Studie zu reflektieren: Fund und Fundumstände, Größe und Anzahl der Samples, Wahl der Vergleichssamples, die ausreichende Menge an Untersuchungsmaterial; ferner, welche umgebenden wissenschaftlichen Ergebnisse wurden berücksichtigt, welche Begriffe werden für welche Zeiten und Räume verwandt? Wurden Zusatzinformationen aus der modernen naturwissenschaftlichbiologischen und der klassischen Archäologie, Linguistik, Ur- und Frühgeschichte herangezogen? Gelten die Vergleichsmerkmale insbesondere zu modernen Vergleichspopulationen?

Angesichts der scheinbaren Evidenz von Maschinen ist in der molecular historiography die Fragestellung ausschlaggebend - die Befunde an sich sagen nichts bis wenig. Erst aus einer Fragestellung und der Validierung der Befunde und des gesamten Untersuchungsdesigns ergibt sich eine Erzählung: Dies können rekonstruierte Individuen sein, dies können rekonstruierte Lebewesen sein, dies können rekonstruierte Dinge sein, dies können 
schließlich in Kartenwerken rekonstruierte Migrationen in Zeit und Raum sein. Auch hier ergibt sich eine neue historische Tatsache erst aus der Wahrnehmung und Kritik der Fachöffentlichkeit - und zwar, soweit möglich, der gesamten Historiographie. Allein die Forschungsgeschichte zum Mann vom Tisenjoch sollte zur Vorsicht mahnen: Alle paar Jahre musste seine Geschichte aufgrund der neuen biologischen Befunde umgeschrieben werden; die lebensweltlichen Umstände seines offenbar gewaltsamen Todes bleiben nach wie vor im Dunkeln. Ein Beispiel, wie weit ein Protagonist seine eigenen Arbeiten kritisch reflektiert, ist Svante Pääbos Frage, ob sich Neandertaler und moderner Mensch vermischt haben. Hinzu kommt, dass sich die Umsatzrate neuer technischer Möglichkeiten einer molecular historiography und damit neuer Befunde stets beschleunigt. Ein Beispiel ist die Populationsgenetik: populationsgenetisch begründete Wanderungsstudien führen je nach technischen Möglichkeiten - Blutgruppen, mtDNA, Y-Chromosomen, aDNA, das gesamte Biom - zu unterschiedlichen Ergebnissen: Die dargestellten Wanderungsbewegungen gelten nicht einem Volk im kulturellen Sinne, sondern ausschließlich der Wanderung der untersuchten genetischen Spezifika. Die daraus resultierenden Aussagen sind extrapoliert. Mit jeder neuen technischen Möglichkeit legen sich gleichsam neue Schichten genetisch nachweisbarer Migrationen übereinander die keinesfalls notwendig mit den zuvor produzierten Karten übereinstimmen müssen. Die kulturhistorische Aussagefähigkeit ergibt sich letztlich nur aus der Kooperation mit anderen Disziplinen: hier etwa der Archäologie oder der Linguistik. So wissen wir jetzt zwar, dass die große Pest des 14. Jahrhunderts tatsächlich eine echte, von Pasteurella/Yersinia pestis verursachte Pest war. In der allgemeinen Historiographie, besonders in der Medizingeschichte sind zur Frage der Natur des Erregers Bibliotheken verfasst worden, die jetzt alle obsolet sind. Gleichwohl hilft die Kenntnis des biologischen Erregers bei den real- und kulturhistorischen Studien der Geschichte jener Zeit wenig.

Was wüssten wir über die Geschichte der Menschheit vor Zeiten der Schriftlichkeit ohne modernste natur- und lebenswissenschaftliche Methoden? Wohl wenig. Was wüssten wir über die Geschichte schriftlicher Zeiten ohne klassische historische Methoden? Auch wenig. So läuft alle wechselseitige Kritik notwendig auf eine Kooperation sowohl der Geistes-, Kultur- und Sozialwissenschaften einerseits und andererseits der Naturund Lebenswissenschaften hinaus. Letztlich geht es darum, die Geschichte keineswegs nur der Menschheit, sondern unseres gesamten Lebensraumes über die gesamte Zeit hin aufzuklären. Eine Geschichte des Anthropozän (Trischler 2016) wird keine einzelne Disziplin allein leisten können. Aus dem kritisch-konstruktiven „Streit der Fakultäten“ wird sich die Reichwei- 
te, mit der die verschiedenen Disziplinen Aussagen treffen können, von selbst ergeben.

\section{Anmerkungen}

1 Vgl. aus den abundanten, durchweg vorzüglich ausgestatteten Monographien der jüngsten Jahre u.v. a.: Pääbo (2014); Parzinger (2015); Parzinger (2016); Cunliffe (2016). Als aktuelle Einführung in die bedeutenden Entwicklungen in der Archäologie Chinas, siehe Labisch (2018).

2 Vgl. Forum Genetic History im vorliegenden Heft; vgl. ferner Bösl, Elsbeth und Jörg Feuchter: Ancient DNA (Arbeitstitel). Neue Politische Literatur (in Vorbereitung); für eine umfassende Übersicht der Forschungsliteratur sei auf das Buch von Elsbeth Bösl (2017a) verwiesen.

3 Vgl. auch Bösl (2017b).

\section{Literatur}

Atzmon, Gil, et al. 2010. Abraham's Children in the Genome Era: Major Jewish Diaspora Populations Comprise Distinct Genetic Clusters with Shared Middle Eastern Ancestry. American Journal of Human Genetics (86): 850-859.

Behar, Doron M., et al. 2010. The Genome-wide Structure of the Jewish People. Nature (466): 238-242.

Bösl, Elsbeth 2017a. Doing Ancient DNA. Zur Wissenschaftsgeschichte der aDNA-Forschung. Bielefeld: Transcript

Bösl, Elsbeth, 2017b. Zur Wissenschaftsgeschichte der aDNA-Forschung. NTM Zeitschrift für Geschichte der Wissenschaften, Technik und Medizin (25), 99-142

Bösl, Elsbeth/Feuchter, Jörg (in Vorbereitung). Ancient DNA (Arbeitstitel). Neue Politische Literatur.

Cunliffe, Barry W. 2016. 10000 Jahre. Geburt und Geschichte Eurasiens. Darmstadt: wbg Theiss.

Labisch, Alfons 2004. Die bakteriologische und die molekulare Transition der Medizin. Historizität und Kontingenz als Erkenntnismittel. In: Labisch, Alfons und Norbert Paul (Hg.). Historizität. Erfahrung und Handeln - Geschichte und Medizin (= Sudhoffs Archiv. Zeitschrift für Wissenschaftsgeschichte. Beihefte, Heft 54). Stuttgart: Steiner: 213-226.

Labisch, Alfons 2018. Eurasian Transfer of Knowledge vs. Eurasian Interchange of Knowledge - The Times Before Writing. Journal for Cultural Interaction in East Asia (9): 31-58.

Müller-Wille, Staffan und Christina Brandt (Hg.) 2016. Heredity Explored. Between Public Domain and Experimental Science, 1850-1930. Boston: The MIT-Press.

Ostrer, Harry und Karl Skorecki 2013. The Population Genetics of the Jewish People. Human Genetics (132): 119-127.

Pääbo, Svante 2014. Neanderthal Man. In Search of Lost Genomes. New York: Basic Books (dt.: Die Neandertaler und wir. Meine Suche nach den Urzeit-Genen. Frankfurt a.M.: S. Fischer, 2014).

Parzinger, Hermann 2015. Die Kinder des Prometheus. Eine Geschichte der Menschheit vor der Erfindung der Schrift. München: Beck.

Parzinger, Hermann 2016. Abenteuer Archäologie. Eine Reise durch die Menschheitsgeschichte. München: Beck. 
Rheinberger, Hans-Jörg 2001. Experimentalsysteme und epistemische Dinge. Eine Geschichte der Proteinsynthese im Reagenzglas. Göttingen: Wallstein

Samida, Stefanie und Jörg Feuchter (Hg.) 2016. The Genetic Challenge to Medieval History and Archaeology. Medieval Worlds (4).

Sommer, Marianne, 2016. History Within. The Science, Culture, and Politics of Bones, Organisms, and Molecules. Chicago: The University of Chicago Press.

Trischler, Helmuth 2016. The Anthropocene. A Challenge for the History of Science, Technology, and the Environment. NTM Zeitschrift für Geschichte der Wissenschaften, Technik und Medizin (24): 309-35.

\author{
Alfons Labisch \\ c/o University Hospital \\ Heinrich-Heine-University Düsseldorf \\ Moorenstraße 5, Bldg. 14.75 \\ 40225 Düsseldorf \\ Deutschland \\ alfons.labisch@hhu.de
}

